\title{
Heavy Quark Masses (from QCD Sum Rules) and their impact on the $(g-2)_{\mu}$
}

\author{
Jens Erler, ${ }^{a+\star}$ Pere Masjuan $^{b, *}$ and Hubert Spiesberger ${ }^{a \|}$ \\ ${ }^{a}$ PRISMA ${ }^{+}$Cluster of Excellence, Institute for Nuclear Physics ${ }^{\star}$ and Institute of Physics ${ }^{\prime}$, Johannes \\ Gutenberg-University, 55099 Mainz, Germany \\ ${ }^{b}$ Grup de Física Teòrica, Departament de Física, Universitat Autònoma de Barcelona, \\ and Institut de Física d'Altes Energies, Barcelona Institute of Science and Technology, Campus UAB, \\ E-08193 Bellaterra (Barcelona), Spain
}

E-mail: erler@uni-mainz.de, masjuan@ifae.es, spiesber@uni-mainz.de

We determine the charm and the bottom quark masses $m_{c}\left(m_{c}\right)$ and $m_{b}\left(m_{b}\right)$ from QCD sum rules of moments of the vector current correlator calculated in perturbative QCD. Only experimental data for the heavy-quark resonances below the continuum threshold are needed in our approach, while the continuum contribution is determined by requiring self-consistency between various sum rules, including the one for the zeroth moment. Existing data from the continuum region can then be used to constraint the theoretical error providing a suitable parameterization of such continuum region. Our result is $m_{c}\left(m_{c}\right)=1272 \pm 8 \mathrm{MeV}$ and $m_{b}\left(m_{b}\right)=4180 \pm 9 \mathrm{MeV}$ for $\alpha_{s}\left(M_{z}\right)=0.1182$. As a byproduct, the parameterization of the $R(s)$ function and the heavy-quark masses are used to determine the contribution of the heavy quarks to the Hadronic Vacuum Polarization contribution to the anomalous magnetic moment of the muon yielding $a_{\mu, L O}^{\text {had, charm }}=14.36(23) \times 10^{-10}$ and $a_{\mu, L O}^{\text {had, bottom }}=0.30(2) \times 10^{-10}$ respectively.

\footnotetext{
*** 10th International Workshop on Charm Physics (CHARM2020), ***

***31 May - 4 June, 2021 ***

*** Mexico City, Mexico - Online ***
}

\footnotetext{
${ }^{*}$ Speaker
} 


\section{Method and Results}

We report on work $[1,2]$ on the determination of the heavy quark mass, $m_{c}\left(m_{c}\right)$ and $m_{b}\left(m_{b}\right)$. Besides being a fundamental input parameter defining the Standard Model (SM), $m_{c}$ and $m_{b}$ enter in many QCD and electroweak processes: from the renormalization group running of the fine structure constant (via the $0^{\text {th }}$ moment sum rule), to the running of the weak mixing angle from the $\mathrm{Z}$ pole down to low energies, including the SM prediction of the anomalous magnetic moment of the muon [3]. An important future application will be the test of the mass versus Yukawa coupling relation in the single Higgs SM, because at future lepton colliders it will be possible to measure the charm and bottom Yukawa couplings very precisely.

One can also determine heavy-quark masses in lattice simulations, but it is prudent to inquire for a second opinion derived from an independent first principles approach. This approach is provided by the relativistic QCD sum rule formalism describing low moments of the heavy quark (q) vector-current correlator $\Pi_{q}$, where the master equation is given by

$$
12 \pi^{2} \frac{\Pi_{q}(0)-\Pi_{q}(-t)}{t}=\int_{4 m_{q}^{2}}^{\infty} \frac{\mathrm{d} s}{s} \frac{R_{q}(s)}{s+t},
$$

where $R_{q}(s)=12 \pi \operatorname{Im} \Pi_{q}(s)$, and where $m_{q}=m_{q}\left(m_{q}\right)$ is the heavy quark mass, $q=c, b$.

The right-hand side is basically an integral over electromagnetic heavy-quark pair production (normalized to the muonic cross-section) with certain weights. $\Pi_{q}$ has been calculated in perturbative QCD to $O\left(\alpha_{s}^{3}\right)$ [4-8]. In the limit $t \rightarrow 0$, the left-hand side of Eq. (1) turns into a derivative with the right-hand side being suppressed by two powers of $s$ and providing the $1^{s t}$ moment, $\mathcal{M}_{1}$. Taking further derivatives generates higher moments, $\mathcal{M}_{n}$ [9]. But one can also take the opposite limit, $t \rightarrow \infty$, which corresponds to the $0^{t h}$ moment sum rule, $\mathcal{M}_{0}[1,3]$, mentioned already before. As it stands, $\mathcal{M}_{0}$ shall be regularized using the asymptotic perturbative expansion, $R_{q}(s) \equiv 4 / 3 \lambda_{1}^{q}(s)$ in the mass-less limit on both sides of the equation.

$\mathcal{M}_{0}$ is one of the ingredients where our analysis differs from others. Another special feature of our approach is that the only experimental input are the electronic widths of the charmonium and bottomonium states, $\Psi(1 S), \Psi(2 S)$ for charm-mass determination and $\Upsilon(1 S), \Upsilon(2 S), \Upsilon(3 S)$ for bottom-mass determination. The continuum contribution is in practice parametrized using the ansatz

$$
R_{q}^{\mathrm{cont}}(s)=3 Q_{q}^{2} \lambda_{1}^{q}(s) \sqrt{1-\frac{4 m_{q}^{2}(2 M)}{s^{\prime}}}\left[1+\lambda_{3}^{q}\left(\frac{2 m_{q}^{2}(2 M)}{s^{\prime}}\right)\right],
$$

where $3 Q_{q}^{2} \lambda_{1}^{q}(s)$ is the zero-mass limit of $R_{q}(s)$ and $s^{\prime}:=s+4\left[m_{q}^{2}(2 M)-M^{2}\right] . \quad M$ is taken as the mass of the lightest pseudoscalar meson, i.e., in our case $M=M_{D^{ \pm}}$and $M=M_{B^{ \pm}}$for charm and bottom respectively. Such continuum which guarantees a smooth transition between the onset of the heavy-quark production threshold at $2 M$ and pQCD at large $s$, will be constrained by requiring self-consistency between various sum rules, allowing to determine the quark mass but also the auxiliary parameter $\lambda_{3}^{q}$. This is where $\mathcal{M}_{1}$ comes in handy because it experiences stronger sensitivity to the continuum compared to higher moments, and at the same time a milder sensitivity 
to the quark mass. With our ansatz, we avoid to perform the integral in Eq. (1) up to some value of $s$ after which no more data are available and where one needs to switch to perturbative QCD, changing from hadron to quark degrees of freedom at this particular energy point, procedure which is not rigorously justified, although common in previous analysis. Finally, in our approach it is also possible to estimate correlated errors across various moments, self-constraining the result. Our final result for the $\bar{M} S$ charm- and bottom-quark mass are:

$$
\begin{gathered}
m_{c}\left(m_{c}\right)=\left(1272+2616 \Delta \alpha_{s} \pm 8\right) \mathrm{MeV} \\
m_{b}\left(m_{b}\right)=\left(4180-109 \Delta \alpha_{s} \pm 8\right) \mathrm{MeV} .
\end{gathered}
$$

We explicitly exhibit the dependence on the input value of the strong coupling $\alpha_{s}$ relative to the central value, i.e., $\Delta \alpha_{s}=\alpha_{s}\left(M_{Z}\right)-0.1182$.

\section{Discussion and phenomenology}

Figure 1 shows the total uncertainty and the error breakdown as a function of the pair of moments considered, $0^{t h}-n^{t h}$. Blue bars represent the full error. The experimental input error from the electronic partial widths is shown in red. For the truncation error (in green) we used a somewhat more conservative estimate than simply taking the last available term. Cyan bars account for the comparison of our method of constraints on the continuum region from internal consistency via Eq.(2) and pairs of moments with actual experimental moments calculated in the energy region for which data are available. This comparison should allow for the possibilities of larger-than-expected duality violations (while the assumption of quark-hadron duality in a finite region is much weaker than local duality, it still lacks complete rigour) or high-order terms in the operator product expansion (OPE), given that specially the charm quark mass is dangerously close to the hadronic scale. Using $e^{+} e \rightarrow$ hadrons electro-production data, the experimental moments allow us to obtain a new $\lambda_{3}^{q \text {,exp }}$ parameter, which due to experimental errors will have an error itself. Cyan bars then are the symmetrized error combinations due to $\lambda_{3}^{q} \neq \lambda_{3}^{q \text {,exp }}$ and $\Delta \lambda_{3}^{q}$. As for the gluon condensate, we take the entire estimated contribution as the uncertainty (in orange). For the bottom case, such contribution is negligible. We also show the parametric uncertainty (in purple) from $\alpha_{s}\left(M_{Z}\right)=0.1182 \pm 0.0016$.

The optimal pair of moments returns the smallest total uncertainty as a trade off from the different error sources, $0^{\text {th }}-2^{\text {nd }}$ for the charm quark and $0^{\text {th }}-7^{\text {th }}$ for the bottom quark. In this last case, adding on top of the continuum the $\Upsilon(4 S)$ and $\Upsilon(5 S)$ helps improving the convergence of the sequence. In both cases, the largest uncertainty comes from the truncation error in the theoretical moments.

A comparison with previous results for heavy-quark masses are collected in Fig.2, for charm (top) from [10-14] and for bottom from [11, 12, 15-28] .

The Hadronic Vacuum Polarization is the largest source of uncertainty in the Standard Model prediction of the anomalous magnetic moment of the muon [29]. To account for it, one needs a full description of the $\sigma_{\text {had }}(s)=\sigma\left(e^{+} e^{-} \rightarrow\right.$ hadrons $)$. Since such information is not available (experimental data covers a limited energy range only), one usually combines data + pQCD[29] 

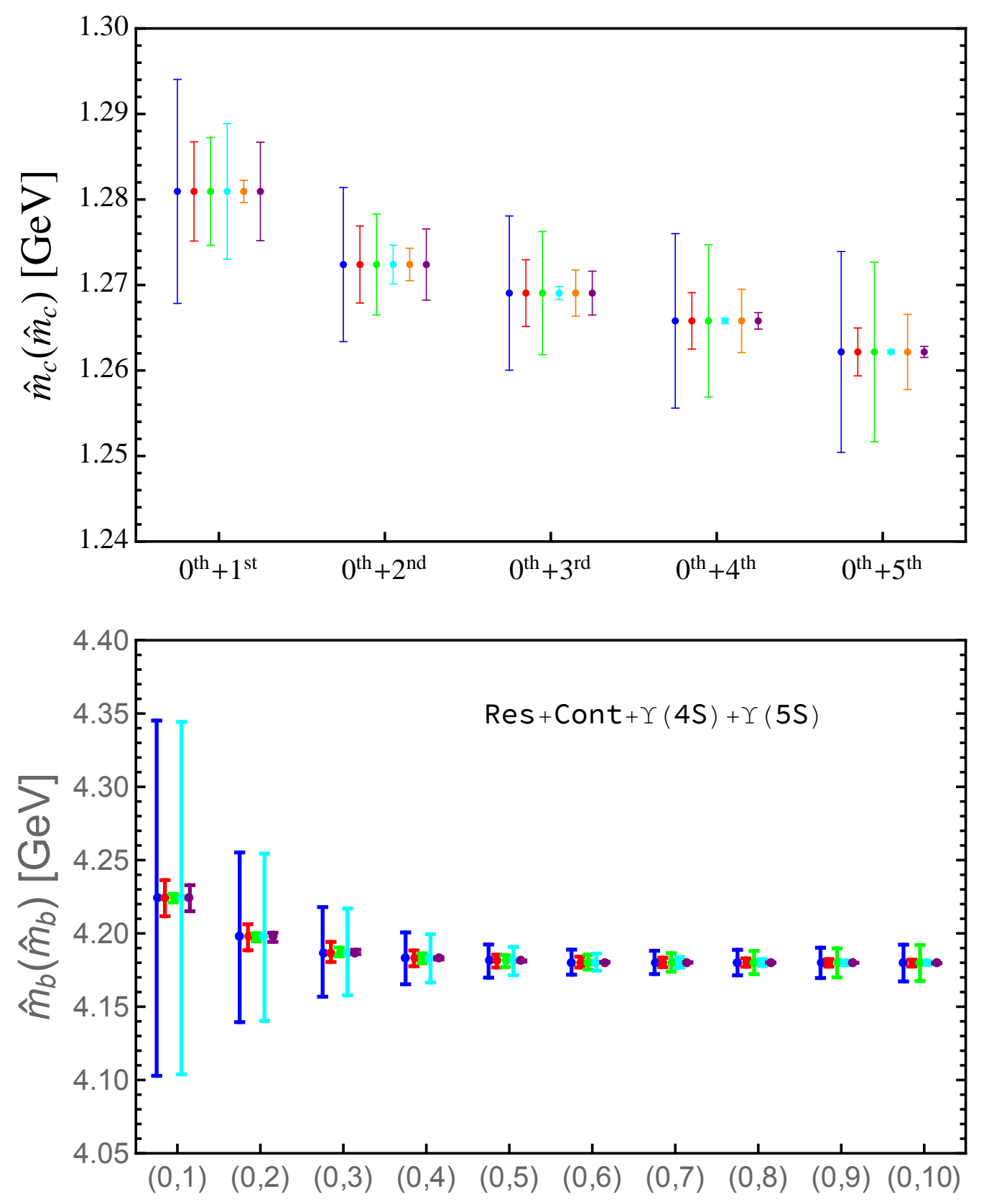

Figure 1: Results for $m_{c}\left(m_{c}\right)$ (top) $m_{b}\left(m_{b}\right)$ (bottom) using different combinations of moments. For the bottom case we added the $\Upsilon(4 S)$ and $\Upsilon(5 S)$ states explicitly to the ansatzin Eq.(2). Blue bars represent the full error, red bars are from the experimental uncertainties in the resonance parameters, green bars indicate the truncation errors in the theoretical moments, cyan bars are the symmetrized error combinations due to $\lambda_{3}^{q} \neq \lambda_{3}^{q \text {,exp }}$ and $\Delta \lambda_{3}^{q}$, orange bars represent the error coming from gluon condensate (negligible for the bottom case) and the uncertainty induced by $\Delta \alpha_{s}\left(M_{Z}\right)= \pm 0.0016$ is shown in purple. 


\begin{tabular}{c|c|c|c|c|c|c|c|} 
& central value & total error & resonances & Truncation & $\Delta \lambda_{q}^{3}$ & Condensates & $\Delta \alpha_{s}$ \\
\hline$a_{\mu, L O}^{\text {had, }}$ & 14.36 & 0.23 & 0.12 & 0.04 & 0.18 & 0.01 & 0.05 \\
$a_{\mu, L O}^{\text {had, b }}$ & 0.30 & 0.02 & 0.00 & 0.00 & 0.02 & - & 0.01
\end{tabular}

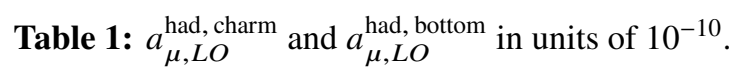

changing hadrons to quarks in a particular energy point. We can now avoid such not rigorously justified approximation using our Eq.(2) provided that both the quark mass and $\lambda_{q}^{3}$ are known. Using our quark-mass results, the following equation provides the desired result:

$$
a_{\mu, L O}^{\mathrm{had}}=\frac{1}{4 \pi^{3}} \int_{m_{\pi^{0}}^{2}}^{\infty} \mathrm{d} s K(s) \sigma_{\mathrm{had}}(s),
$$

where $K(s)$ is a kernel function dealing with kinematics [29], and $\sigma_{\text {had }}(s)=\frac{4 \pi \alpha_{\mathrm{em}}^{2} R(s)}{3 s}$. For charm and bottom we find:

$$
\begin{gathered}
a_{\mu, L O}^{\text {had, charm }}=14.36(23) \times 10^{-10}, \\
\quad a_{\mu, L O}^{\text {had, bottom }}=0.30(2) \times 10^{-10} .
\end{gathered}
$$

where the error budget is accounted for in Table 1 , yielding $a_{\mu, L O}^{\text {had, heavy quarks }}=14.66(23) \times 10^{-10}$ with top-quark contribution negligible at that precision.

\section{Acknowledgments}

This work was supported by the German-Mexican research collaboration grant No. 278017 (CONACyT) and No. SP 778/4-1 (DFG). P.M has received funding from the Spanish Ministry of Science and Innovation (PID2020-112965GB-I00/AEI/ 10.13039/501100011033) and from the Agency for Management of University and Research Grants of the Government of Catalonia (project SGR 1069).

\section{References}

[1] J. Erler, P. Masjuan and H. Spiesberger, Eur. Phys. J. C 77, 99 (2017). arXiv:1610.08531 [hep-ph]

[2] J. Erler, P. Masjuan and H. Spiesberger, In preparation.

[3] J. Erler and M. Luo, Phys. Lett. B 558, 125 (2003). arXiv:hep-ph/0207114 [hep-ph]

[4] K. G. Chetyrkin, J. H. Kühn and C. Sturm, Eur. Phys. J. C 48, 107 (2006). arXiv:hep$\mathrm{ph} / 0604234$ [hep-ph]

[5] R. Boughezal, M. Czakon and T. Schutzmeier, Phys. Rev. D 74, 074006 (2006). arXiv:hepph/0605023 [hep-ph] 

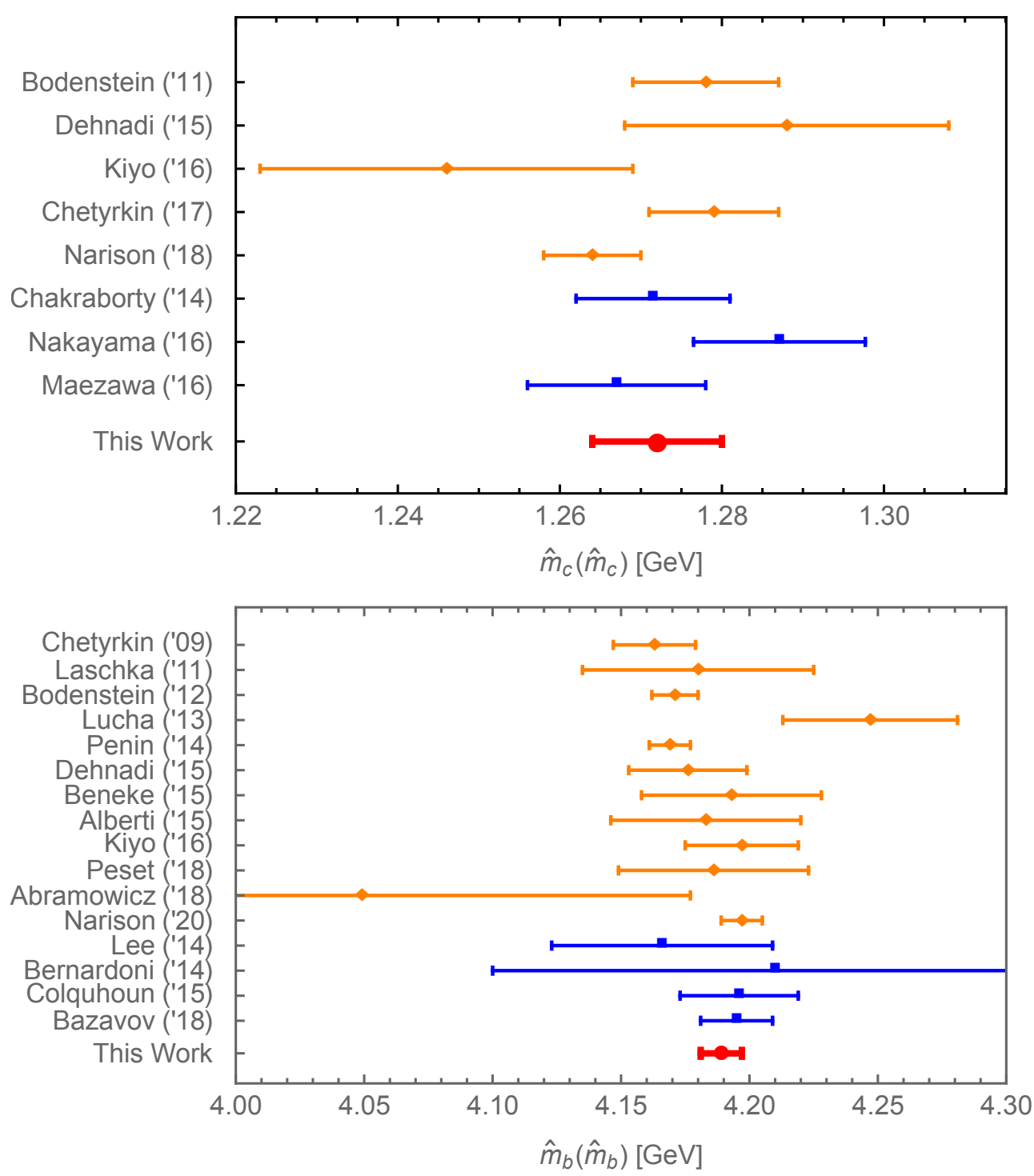

Figure 2: Recent charm (top) and bottom (bottom) quark mass determinations from phenomenological studies (orange diamonds) and lattice calculations (blue squares).

[6] B. A. Kniehl and A. V. Kotikov, Phys. Lett. B 642, 68 (2006). arXiv:hep-ph/0607201 [hep-ph]

[7] A. Maier, P. Maierhofer and P. Marquard, Phys. Lett. B 669, 88 (2008). arXiv:0806.3405 [hep-ph]

[8] D. Greynat, P. Masjuan and S. Peris, Phys. Rev. D 85, 054008 (2012). arXiv:1104.3425 [hep-ph]

[9] V. A. Novikov et al, Phys. Rept. 41, 1 (1978). doi:10.1016/0370-1573(78)90120-5

[10] S. Bodenstein, J. Bordes, C. A. Dominguez, J. Penarrocha, and K. Schilcher. Phys. Rev. D, 83, $074014,2011$. 
[11] B. Dehnadi, A. H. Hoang and V. Mateu, JHEP 08, 155 (2015). arXiv:1504.07638 [hep-ph]

[12] Y. Kiyo, G. Mishima and Y. Sumino, Phys. Lett. B 752 (2016), 122-127 [erratum: Phys. Lett. B 772 (2017), 878-878] doi:10.1016/j.physletb.2015.11.040 [arXiv:1510.07072 [hep-ph]].

[13] K. G. Chetyrkin, J. H. Kuhn, A. Maier, P. Maierhofer, P. Marquard, M. Steinhauser and C. Sturm, Phys. Rev. D 96 (2017) no.11, 116007 [arXiv:1710.04249 [hep-ph]].

[14] S. Narison, Phys. Lett. B 784 (2018) 261 [arXiv:1808.01969 [hep-ph]].

[15] K. G. Chetyrkin et al., Phys. Rev. D 80, 074010 (2009). arXiv:0907.2110 [hep-ph]

[16] A. Laschka, N. Kaiser and W. Weise, Phys. Rev. D 83 (2011), 094002 [arXiv:1102.0945 [hep-ph]].

[17] S. Bodenstein, J. Bordes, C. A. Dominguez, J. Penarrocha and K. Schilcher, Phys. Rev. D 85 (2012), 034003 [arXiv:1111.5742 [hep-ph]].

[18] W. Lucha, D. Melikhov and S. Simula, Phys. Rev. D 88 (2013), 056011 [arXiv:1305.7099 [hep-ph]].

[19] A. A. Penin and N. Zerf, JHEP 04 (2014), 120 [arXiv:1401.7035 [hep-ph]].

[20] M. Beneke, A. Maier, J. Piclum and T. Rauh, Nucl. Phys. B 891 (2015), 42-72 [arXiv:1411.3132 [hep-ph]].

[21] A. Alberti, P. Gambino, K. J. Healey and S. Nandi, Phys. Rev. Lett. 114 (2015) no.6, 061802 [arXiv:1411.6560 [hep-ph]].

[22] C. Peset, A. Pineda and J. Segovia, JHEP 09 (2018), 167 [arXiv:1806.05197 [hep-ph]].

[23] H. Abramowicz et al. [H1 and ZEUS], Eur. Phys. J. C 78 (2018) no.6, 473 [arXiv:1804.01019 [hep-ex]].

[24] S. Narison, Phys. Lett. B 802 (2020), 135221 [arXiv:1906.03614 [hep-ph]].

[25] A. J. Lee et al. [HPQCD], Phys. Rev. D 87 (2013) no.7, 074018 [arXiv:1302.3739 [hep-lat]].

[26] F. Bernardoni, B. Blossier, J. Bulava, M. Della Morte, P. Fritzsch, N. Garron, A. Gerardin, J. Heitger, G. von Hippel and H. Simma, et al. Phys. Lett. B 730 (2014), 171-177 [arXiv:1311.5498 [hep-lat]].

[27] B. Colquhoun, R. J. Dowdall, C. T. H. Davies, K. Hornbostel and G. P. Lepage, Phys. Rev. D 91 (2015) no.7, 074514 [arXiv:1408.5768 [hep-lat]].

[28] A. Bazavov et al. [Fermilab Lattice, MILC and TUMQCD], Phys. Rev. D 98 (2018) no.5, 054517 [arXiv:1802.04248 [hep-lat]].

[29] T. Aoyama, N. Asmussen, M. Benayoun, J. Bijnens, T. Blum, M. Bruno, I. Caprini, C. M. Carloni Calame, M. Cè and G. Colangelo, et al. Phys. Rept. 887 (2020), 1-166 [arXiv:2006.04822 [hep-ph]]. 